\title{
COMMENTARY
}

\section{Ethyl pyruvate for the treatment of acetaminophen intoxication: alternative to $\mathrm{N}$-acetylcysteine?}

\author{
Florian Wagner', Pierre Asfar², Michael Georgieff', Peter Radermacher ${ }^{1 *}$ and Katja Wagner' \\ See related research by Yang et al., http://ccforum.com/content/16/1/R9
}

\begin{abstract}
$\mathrm{N}$-acetylcysteine is the classical antidote for acetaminophen overdose-induced hepatotoxicity, but its efficacy is limited by the need for early and only temporary treatment. Therefore, Yang and colleagues tested the hypothesis of whether ethyl pyruvate another anti-inflammatory and antioxidant compound, which they had previously shown to protect against liver injury of various other etiologies - may allow circumventing these limitations. While ethyl pyruvate improved liver regeneration when administered early and during a limited period only, the opposite response was present both after delayed as well as prolonged treatment. The authors concluded that prolonged anti-inflammatory treatment is detrimental after acetaminophen intoxication-induced liver damage. On the one hand, this research paper confirms the need for biomarkers to monitor organ recovery after acetaminophen. On the other hand, this paper adds to the ongoing discussion on the usefulness of ethyl pyruvate as a resuscitation fluid in the critically ill.
\end{abstract}

In the present issue of Critical Care, Yang and colleagues report on the effects of ethyl pyruvate (EP) during acetaminophen intoxication-induced hepatotoxicity [1]. Two hours after an acetaminophen challenge, mice received vehicle or EP every 8 hours for a total of 24 or 48 hours. In a fourth experiment, treatment was started at 24 hours post acetaminophen only. The major findings were that EP attenuated the biochemical and histological markers of liver damage when the treatment was initiated early

*Correspondence: peter.radermacher@uni-ulm.de

'Sektion Anästhesiologische Pathophysiologie und Verfahrensentwicklung, Klinik für Anästhesiologie, Universitätsklinikum, Helmholtzstrasse 8-1, D-89081 Ulm, Germany

Full list of author information is available at the end of the article and maintained over 24 hours only, and that both delayed and prolonged treatments even aggravated organ injury. Interestingly, prolonged treatment coincided not only with decreased expression of cellular cyclin $\mathrm{D}_{1}$, a marker of liver cell regeneration, but also with reduced serum levels of TNFa. The authors conclude that despite the well-established beneficial effect of early and anti-inflammatory and antioxidant therapy after acetaminophen intoxication, prolonged treatment is likely to impair hepatocyte regeneration.

Why did the authors choose EP? First, the main author's group had previously demonstrated that EP protected against liver injury resulting from alcohol overdose, bile duct ligation-induced cholestasis, and acute pancreatitis, thus confirming its beneficial effects in murine shock states - for example, hemorrhage [2] or sepsis [3,4]. Second, acetaminophen intoxication-induced hepatoxicity results from the formation of the toxic metabolite $N$-acetyl- $p$-benzoquinone imine, which causes severe oxidative stress-related cell damage due to depletion of glutathione. The glutathione precursor $\mathrm{N}$-acetylcysteine (NAC) is therefore currently the antidote of choice for the treatment of acetaminophen overdose. However, the authors had previously demonstrated that prolonged NAC administration aggravated liver damage and impaired hepatocellular regeneration [5]. Consequently, they now tested a compound with comparable anti-inflammatory and antioxidant properties. In fact, beside its role as an intermediate glucose metabolite, pyruvate per se is well established as an oxygen radical scavenger with anti-inflammatory properties, resulting from the inhibition of TNF $\alpha$ and IL- 6 production, the attenuated activation of NF- $\mathrm{KB}$, cyclooxygenase- 2 and the inducible isoform of nitric oxide synthase, and the reduction of circulating high-mobility group protein B-1 levels [6,7]. Pyruvate, however, is unstable in aqueous solutions due to a rapid aldol-like condensation reaction forming parapyruvate, a potent inhibitor of the Krebs cycle blocking the $\alpha$-ketoglutaric dehydrogenase [7]. This problem can be circumvented by using the ethanol ester of pyruvate (that is, EP) - the effects of which qualitatively resemble that of pyruvate, but which only require 
molar doses that are lower by one to two orders of magnitude [7]. In addition to the properties of pyruvate, EP also exerts anti-apoptotic effects, increases heme oxygenase-1 expression, attenuates peroxynitrite-related DNA damage, and stabilizes the hypoxia-inducible factor $1 \alpha$ via stimulation of the Krebs cycle.

What do we learn from the study by Yang and colleagues? The authors confirm their previous findings that some degree of inflammation seems to be crucial for hepatocyte regeneration after acetaminophen intoxication. Unfortunately, the mechanisms responsible for their observation remain unclear: no information on highmobility group protein B-1 concentrations is provided, one of the major targets of EP [3,7], which also assumes particular importance for acetaminophen hepatotoxicity [8]. In their previous study, the authors showed that the impaired liver cell recovery resulting from prolonged NAC treatment was associated with attenuated NF- $\mathrm{kB}$ activation [5]. There are no data on NF- $\mathrm{KB}$ activation either, and the authors therefore missed the opportunity of further elucidating the friend and foe character of NF- $\kappa B$ activation and its modulation by EP, respectively, in shock states. Su and colleagues showed in murine endotoxemia that EP doses ranging from 0.01 to $100 \mathrm{mg} /$ $\mathrm{kg}$ caused an early (at 3 hours) decrease and a subsequent late (at 9 hours) moderate increase of lung and kidney NF- $\mathrm{KB}$ activation ultimately associated with higher mortality [9]. Furthermore, the second major property of EP - that is, its role as an antioxidant and, consequently, its impact on oxidative stress - remains a matter of debate. The authors did not find any intergroup differences for tissue malondialdehyde concentrations, a marker of lipid peroxidation, but the activities of antioxidant enzymes (for example, superoxide dismutase, catalase, glutathione peroxidase) were not assessed. Finally, there are no data on glutathione depletion, which, however is referred to as the key mechanism of acetaminophen hepatotoxicity.

Which conclusions can be drawn from the study by Yang and colleagues? The authors have the merit of highlighting the time and dose dependency of any antioxidant administration for acetaminophen intoxication, thus confirming the need for biomarkers that would allow monitoring of liver regeneration and, thus, determination of the tipping point when treatment should be withdrawn [10]. Moreover, the authors add another piece to the puzzling fate of EP in the treatment of the critically ill. Clearly, it is a tempting perspective to have a resuscitation fluid with a 'twist of an anti-inflammatory antioxidant' [11], but the authors' current and previous studies on NAC are exemplary for the equivocal effects of antioxidants - that is, the antioxidant paradox [12]: depending on transition metal release, any non-enzymatic antioxidant can become a pro-oxidant and thereby worsen damage. Previously, Magder elegantly highlighted this dilemma: depending on their type, cellular source, and biological target, 'we need to regulate reactive oxygen species, not eradicate them' [13]. In this context, the role of EP is still not well defined: despite the unanimous data from (mostly unresuscitated) rodent models, the findings in clinically more relevant, resuscitated large animal models are controversial - both beneficial effects [14-16] and lacking efficacy $[17,18]$ were reported. The only clinical study available demonstrated that EP was safe when administered after induction of anesthesia for highrisk cardiac surgery, but did not confer any benefit with respect to mortality and morbidity as assessed by the duration of mechanical ventilation, the incidence of acute kidney injury, or the need for hemodynamic support [19].

\section{Abbreviations}

EP, ethyl pyruvate; IL, interleukin; NAC, N-acetylcysteine; NF, nuclear transcription factor; TNF, tumor necrosis factor.

\section{Competing interests}

The authors declare that they have no competing interests.

\section{Author details}

'Sektion Anästhesiologische Pathophysiologie und Verfahrensentwicklung, Klinik für Anästhesiologie, Universitätsklinikum, Helmholtzstrasse 8-1, D-89081 UIm, Germany. 'Laboratoire HIFIH, UPRES EA 3859, IFR 132, Université d'Angers, Département de Réanimation Médicale et de Médecine Hyperbare, Centre Hospitalo-Universitaire, 4 rue Larrey, 49933 Angers Cedex 09, France.

Published: 20 February 2012

\section{References}

1. Yang R, Zou X, Koskinen ML, Tenhunen J: Ethyl pyruvate reduces liver injury at early phase but impairs regeneration at late phase in acetaminophen overdose. Crit Care 2012, 16:R9.

2. Yang R, Gallo DJ, Baust JJ, Uchiyama T, Watkins SK, Delude RL, Fink MP: Ethyl pyruvate modulates inflammatory gene expression in mice subjected to hemorrhagic shock. Am J Physiol Gastrointest Liver Physiol 2002, 283:G212-G221.

3. Ulloa L, Ochani M, Yang H, Tanovic M, Halperin D, Yang R, Czura CJ, Fink MP, Tracey KJ: Ethyl pyruvate prevents lethality in mice with established lethal sepsis and systemic inflammation. Proc Natl Acad Sci U S A 2002, 99:12351-12356.

4. Miyaji T, Hu X, Yuen PST, Muramatsu Y, lyer S, Hewitt SM, Star RA: Ethyl pyruvate decreases sepsis-induced acute renal failure and multiple organ damage in aged mice. Kidney Int 2003, 64:1620-1631.

5. Yang R, Miki K, He X, Killeen ME, Fink MP: Prolonged treatment with $\mathrm{N}$-acetylcysteine delays liver recovery from acetaminophen hepatotoxicity. Crit Care 2009, 13:R55.

6. Das UN: Pyruvate is an endogenous anti-inflammatory and anti-oxidant molecule. Med Sci Monit 2006, 12:RA79-RA84.

7. Kao KK, Fink MP: The biochemical basis for the anti-inflammatory and cytoprotective actions of ethyl pyruvate and related compounds. Biochem Pharmacol 2010, 80:151-159.

8. Antoine DJ, Williams DP, Kipar A, Jenkins RE, Regan SL, Sathish JG, Kitteringham NR, Park BK: High-mobility group box-1 protein and keratin-18, circulating serum proteins informative of acetaminopheninduced necrosis and apoptosis in vivo. Toxicol Sci 2009, 112:521-531.

9. Su J, Li X, Cui X, Li Y, Fitz Y, Hsu L, Mani H, Quezado M, Eichacker PQ: Ethyl pyruvate decreased early nuclear factor- $\mathrm{KB}$ levels but worsened survival in lipopolysaccharide-challenged mice. Crit Care Med 2008, 36:1059-1067.

10. Nimmi T, Athuraliya C, Jones AL: Prolonged $N$-acetylcysteine therapy in late acetaminophen poisoning associated with acute liver failure - a need to be more cautious? Crit Care 2009, 13:144.

11. Delude RL: Would you like your colloid straight or with a twist of an anti-inflammatory antioxidant? Crit Care Med 2009, 37:1133-1134. 
12. Halliwell B: The antioxidant paradox. Lancet 2000, 355:1179-1180.

13. Magder S: Reactive oxygen species: toxic molecules or spark of life? Crit Care 2006, 10:208.

14. Hauser B, Kick J, Asfar P, Ehrmann U, Albicini M, Vogt J, Wachter U, Brückner UB, Fink MP, Radermacher P, Bracht H: Ethyl pyruvate improves systemic and hepato-splanchnic hemodynamics and prevents lipid peroxidation in a porcine model of resuscitated hyperdynamic endotoxemia. Crit Care Med 2005, 33:2034-2042.

15. Su F, Wang Z, Cai Y, Remmelink M, Vincent JL: Beneficial effects of ethyl pyruvate in septic shock from peritonitis. Arch Surg 2007, 142:166-171.

16. Dong WH, Cai B, Peña G, Pisarenko V, Vida G, Doucet D, Lee M, Sharpe S, Lu Q, Xu DZ, Ramos L, Deitch EA, Ulloa L: Ethyl pyruvate prevents inflammatory responses and organ damage during porcine hemorrhagic shock. Shock 2010, 34:205-213.

17. Mulier KE, Beilman GJ, Conroy MJ, Taylor JH, Skarda DE, Hammer BE: Ringer's ethyl pyruvate in hemorrhagic shock and resuscitation does not improve early hemodynamics or tissue energetics. Shock 2005, 23:248-252.
18. Andersson A, Fenhammar J, Frithiof R, Sollevi A, Hjelmqvist $H$ : Haemodynamic and metabolic effects of resuscitation with Ringer's ethyl pyruvate in the acute phase of porcine endotoxaemic shock. Acto Anaesthesiol Scand 2006, 50:1198-1206.

19. Bennett-Guerrero E, Swaminathan M, Grigore AM, Roach GW, Aberle LG, Johnston JM, Fink MP: A phase II multicenter double-blind placebocontrolled study of ethyl pyruvate in high-risk patients undergoing cardiac surgery with cardiopulmonary bypass. J Cardiothorac Vasc Anesth 2009, 23:324-329.

doi:10.1186/cc11153

Cite this article as: Wagner $F$, et al.: Ethyl pyruvate for the treatment of acetaminophen intoxication: alternative to $\mathrm{N}$-acetylcysteine? Critical Care 2012, 16:112 